\title{
Flavin-Containing Monooxygenase 1 Catalyzes the Production of Taurine from Hypotaurine ${ }^{\mathrm{S}}$
}

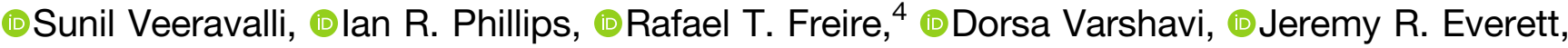 \\ and $\odot$ Elizabeth A. Shephard
}

\begin{abstract}
Department of Structural and Molecular Biology, University College London, London, United Kingdom (S.V., I.R.P., E.A.S.); School of Biological and Chemical Sciences, Queen Mary University of London, London, United Kingdom (I.R.P.); and Medway Metabonomics Research Group, University of Greenwich, Chatham Maritime, Kent, United Kingdom (R.T.F., D.V., J.R.E.)
\end{abstract}

Received November 15, 2019; accepted February 3, 2020

\section{ABSTRACT}

Taurine is one of the most abundant amino acids in mammalian tissues. It is obtained from the diet and by de novo synthesis from cysteic acid or hypotaurine. Despite the discovery in 1954 that the oxygenation of hypotaurine produces taurine, the identification of an enzyme catalyzing this reaction has remained elusive. In large part, this is due to the incorrect assignment, in 1962, of the enzyme as an NAD-dependent hypotaurine dehydrogenase. For more than 55 years, the literature has continued to refer to this enzyme as such. Here we show, both in vivo and in vitro, that the enzyme that oxygenates hypotaurine to produce taurine is flavin-containing monooxygenase (FMO) 1. Metabolite analysis of the urine of Fmo1null mice by ${ }^{1} \mathrm{H}$ NMR spectroscopy revealed a buildup of hypotaurine and a deficit of taurine in comparison with the concentrations of these compounds in the urine of wild-type mice. In vitro assays confirmed that human FMO1 catalyzes the conversion of hypotaurine to taurine, utilizing either NADPH or NADH as cofactor. FMO1 has a wide substrate range and is best known as a xenobiotic- or drugmetabolizing enzyme. The identification that the endogenous molecule hypotaurine is a substrate for the FMO1-catalyzed production of taurine resolves a long-standing mystery. This finding should help establish the role FMO1 plays in a range of biologic processes in which taurine or its deficiency is implicated, including conjugation of bile acids, neurotransmitter, antioxidant and anti-inflammatory functions, and the pathogenesis of obesity and skeletal muscle disorders.

\section{SIGNIFICANCE STATEMENT}

The identity of the enzyme that catalyzes the biosynthesis of taurine from hypotaurine has remained elusive. Here we show, both in vivo and in vitro, that flavin-containing monooxygenase 1 catalyzes the oxygenation of hypotaurine to produce taurine.

\section{Introduction}

Taurine (2-aminoethanesulfonic acid) is one of the most abundant amino acids in mammalian tissues (Huxtable, 1992). It is an organic osmolyte involved in cell volume regulation (Huxtable, 1992) and has a variety of cytoprotective and developmental roles, particularly in neurologic and ocular tissues (Ripps and Shen, 2012). It is also involved in the formation of bile salts (Huxtable, 1992) and the modulation of intracellular calcium concentration (Lombardini, 1983). Taurine is considered an essential substance in mammals, and its deficiency has been implicated in several pathologic conditions.

Taurine is obtained from the diet and, by de novo synthesis, from cysteic acid (Edgar et al., 1994) or hypotaurine (Cavallini et al., 1954). Hypotaurine is itself an organic osmolyte and cytoprotective agent (Yancey, 2005) and acts as an antioxidant to scavenge highly reactive hydroxyl radicals (Aruoma et al., 1988). The oxygenation of

${ }^{4}$ Current affiliation: Signal and Information Processing for Sensing Systems, Institute for Bioengineering of Catalonia (IBEC), The Barcelona Institute of Science and Technology, Barcelona, Spain.

An earlier version of this paper appears in bioRxiv under the doi:1101/750273. https://doi.org/10.1124/dmd.119.089995.

$\$$ This article has supplemental material available at dmd.aspetjournals.org. hypotaurine to produce taurine was discovered in 1954 (Cavallini et al., 1954). Later, the enzyme that converts hypotaurine to taurine was reported to be a NAD-dependent hypotaurine dehydrogenase (Sumizu, 1962) but was not isolated or characterized. Subsequently, Oja and Kontro (1981) noted that the production of taurine in tissue extracts was optimal at pH 9.0 and was stimulated by oxygenation. These authors concluded that the enzyme that converts hypotaurine to taurine was not a NAD-dependent hypotaurine dehydrogenase. By overlooking the work of Oja and Kontro (1981), and giving credence to the earlier study (Sumizu, 1962), the enzyme that catalyzes the conversion of hypotaurine to taurine has continued to be reported as a hypotaurine dehydrogenase (EC 1.8.1.3), utilizing NAD as cofactor. Because an enzyme that catalyzes the reaction has not been identified or isolated, the conversion of hypotaurine to taurine has sometimes been referred to as nonenzymatic.

Here, we show in vivo through the use of a knockout-mouse line and in vitro by assays of human enzymes that oxygenation of hypotaurine to produce taurine is catalyzed by flavin-containing monooxygenase (FMO) 1 .

\section{Materials and Methods}

Animals. All mice used in this study were bred at University College London. The $\mathrm{Fmol}^{-/-}, 2^{-/-}, 4^{-/-}$, and $\mathrm{Fmo5}^{-/-}$mouse lines were constructed as

ABBREVIATIONS: 1D, one dimensional; ca circa FMO, flavin-containing monooxygenase; HMBC heteronuclear multiple bond correlation spectroscopy HSQC, heteronuclear single quantum coherence; TSP, sodium 3-(trimethylsilyl)-2,2',3,3'-tetradeuteropropionate. 
previously described (Hernandez et al., 2006a, 2009; Gonzalez Malagon et al., 2015). Mice were genotyped as described (Hernandez et al. 2006b; Gonzalez Malagon et al., 2015). Mice were given free access to food (a standard chow diet, Teklad Global 18\% ProteinRodent Diet; Harlan Laboratories, Inc., Madison, WI) and water. Animal procedures were carried out in accordance with the UK Animal Scientific Procedures Act as well as with local ethics committee approval (Animal Welfare and Ethical Review Body) and appropriate Home Office Licenses. Urine was collected between 10:00 AM and 12:00 PM (noon) from male and female mice aged 15 to 16 weeks. Urine samples were immediately frozen on solid $\mathrm{CO}_{2}$ and stored at $-80^{\circ} \mathrm{C}$ until analyzed by ${ }^{1} \mathrm{H}$ NMR spectroscopy, as described below.

Enzyme Assays. All reaction mixtures (final volumes of $250 \mu \mathrm{l}$ in a Corning Costar 96-well cell-culture plate; VWR, Lutterworth, Leicestershire, UK) were incubated in a Sunrise absorbance microplate reader (Tecan, Grödig, Austria) equipped with Magellan software, version 6.2. Reaction mixtures contained either $0.1 \mathrm{M}$ potassium phosphate buffer, $\mathrm{pH} 7.4$, or $0.1 \mathrm{M}$ Tris- $\mathrm{HCl}, \mathrm{pH} 8.5,1 \mathrm{mM}$ EDTA (aerated immediately before use by shaking for 5 minutes at room temperature); $2.5 \mathrm{mM}$ hypotaurine (Santa Cruz Biotechnology, Heidelberg, Germany); $0.5 \mathrm{mM}$ NADPH, NADH, or NAD or no cofactor; and baculosomes containing human FMO1, FMO3, or FMO5 (135 nM final concentration) (Sigma Aldrich, Gillingham, Dorset, UK) or an equivalent amount of control insect cell microsomes (Corning Life Sciences, Woburn, MA). The mixtures were incubated at $37^{\circ} \mathrm{C}$ for 60 minutes. Monitoring of NADPH or NADH depletion, measured at $\mathrm{A}_{340 \mathrm{~nm}}$, revealed that reaction velocities were linear over the 60-minute period. Samples were stored at $-80^{\circ} \mathrm{C}$ until analyzed by one-dimensional (1D) ${ }^{1} \mathrm{H}$ NMR spectroscopy, as described below.

Methimazole $S$-oxygenation was measured by the method of Dixit and Roche (1984). Reaction mixtures contained final concentrations of $67 \mathrm{nM}$ of human FMO1 or FMO3 in baculosomes (Sigma Aldrich), $2.5 \mathrm{mM}$ methimazole, and $0.5 \mathrm{mM}$ NADPH or NADH and were incubated at $37^{\circ} \mathrm{C}$.

For estimation of kinetic parameters, reaction mixtures were assembled by adding the following to final concentrations in order: $0.1 \mathrm{M}$ Tris- $\mathrm{HCl}(\mathrm{pH} 8.5)$, $1 \mathrm{mM}$ EDTA (aerated immediately before use by shaking at $37^{\circ} \mathrm{C}$ for 10 minutes), $0.5 \mathrm{mM}$ NADPH, and baculosomes containing human FMO1 (135 nM final concentration) (Sigma Aldrich). The mixtures were equilibrated at $37^{\circ} \mathrm{C}$ for 3 minutes to allow formation of the active $\mathrm{C} 4 \mathrm{a}$-hydroperoxyflavin species of the FAD prosthetic group of FMO. Reactions were initiated by addition of hypotaurine to final concentrations of $1-10 \mathrm{mM}$, or, in the case of blank samples, an equivalent volume of buffer was added. Reaction mixtures were incubated at $37^{\circ} \mathrm{C}$. The initial velocity of enzyme-catalyzed reactions was assessed by monitoring the depletion of NADPH, measured at $\mathrm{A}_{340 \mathrm{~nm}} \Delta \mathrm{A}_{340 \mathrm{~nm}}$ was converted to $\Delta$ NADPH using a molar extinction coefficient of $6.2 \times 10^{3} \mathrm{M}^{-1}$ $\mathrm{cm}^{-1}$ and a light-path length of $0.73 \mathrm{~cm}$. To determine substrate (hypotaurine)dependent oxygenation of NADPH, readings from a blank sample (a reaction mixture containing no hypotaurine) were subtracted.

The ability of hypotaurine to act as a competitor substrate of FMO1 was assessed by measuring the effect of various concentrations of hypotaurine on FMO1-catalyzed $S$-oxygenation of methimazole. Methimazole $S$-oxygenation was measured by the method of Dixit and Roche (1984), as described above, in reaction mixtures containing final concentrations of $67 \mathrm{nM}$ human FMO1 in baculosomes (Sigma Aldrich), $4 \mu \mathrm{M}$ methimazole, and $2.5-100 \mathrm{mM}$ hypotaurine.

Sample Preparation for NMR Spectroscopy. Urine samples $(50 \mu l)$ were prepared for NMR spectroscopy as previously described (Varshavi et al., 2018). Enzyme assay samples were thawed and vortexed, and then $160 \mu 1$ sample was mixed with $80 \mu 10.6 \mathrm{M}$ phosphate buffer, as previously described (Varshavi et al., 2018). The samples were revortexed and centrifuged at 13,000 $\mathrm{g}$ for 5 minutes at $4^{\circ} \mathrm{C}$. Supernatant $(200 \mu \mathrm{l})$ was then pipetted into $3.0-\mathrm{mm}$ outer diameter SampleJet NMR tubes (S-3.0-500-1; Norell).

NMR Spectroscopic Analysis. ${ }^{1} \mathrm{H}$ NMR spectra of urine and enzyme assay samples were recorded on a Bruker Avance III spectrometer (Bruker BioSpin $\mathrm{GmbH}$, Rheinstetten, Germany) operating at $600.44 \mathrm{MHz}$ and at a temperature of $300.0 \mathrm{~K}$, using a standard 1D nuclear Overhauser spectroscopy (NOESY) presaturation pulse sequence with gradient pulses (noesygppr1d), as previously described (Varshavi et al., 2018).

NMR spectral processing was carried out in MNova (MestReNova, version 12.0.1-20560; Mestrelab Research S.L.). All concentrations or ratios of metabolites were calculated using NMR spectroscopy peak areas determined in
MNova. In cases in which the signals were clear of overlap, the areas were calculated without deconvolution. In cases in which there was some peak overlap, the relevant peak areas were calculated after deconvolution, without compromise to the quantification. The following peaks did not require deconvolution: sodium 3-(trimethylsilyl)-2,2',3,3'-tetradeuteropropionate (TSP), NMR reference standard, at 0.0; NAD at circa (ca) 9.35 and ca 9.14; and NADP at ca 9.31 and 9.10. The following peaks required deconvolution prior to area measurement: taurine at ca 3.42 and ca 3.28 ; hypotaurine at ca 3.35 and ca 2.67 ; NADPH at ca 2.85 and ca 2.75 and NADH at ca 2.81 and ca $2.69 \mathrm{ppm}$. Absolute concentrations were determined relative to the known concentration of TSP in the phosphate buffer (1.2 $\mathrm{mg}$ in $11.12 \mathrm{ml}$ ), taking into account that the area of the TSP signal is due to nine protons, whereas other signals, such as those from taurine, are each due to two protons.

The deconvolution of the peaks for metabolite quantification was done using the MNova global spectral deconvolution algorithm. The peak areas were obtained, and the residuals were manually minimized by adjusting the fitting parameters of each peak. Data were imported into Matlab (R2014b; MathWorks).

High-sensitivity analysis by ${ }^{1} \mathrm{H}$ NMR spectroscopy showed that the hypotaurine used as a substrate for enzyme assays contained 0.23 mol percent taurine (area measurement of taurine signal at ca 3.42 relative to the $0.55 \%$ area of the ${ }^{13} \mathrm{C}$ satellite of the hypotaurine signal at ca $3.35 \mathrm{ppm}$ ), and this was taken into account in our calculations.

NMR Data Deposition. Original NMR data will be deposited in MetaboLights (EBI UK) (Haug et al., 2013) after publication.

Metabolite Identification. NMR-based metabolite identification was carried out by using standard methods, as described (Dona et al., 2016), and by using information from the literature and public databases, including the Human Metabolite Database (Wishart et al., 2018) (http://www.hmdb.ca/).2018) (see Supplemental Data). Hypotaurine in $\mathrm{Fmol}^{-/-}, 2^{-/-}$, and $4^{-/-}$mouse urine showed the following features: $2.665(\mathrm{t}, 6.9 \mathrm{~Hz}$ ), 58.6 (HSQC) with HMBC to 36.4 and correlated spectroscopy (see Supplemental Fig. 1 ) to 3.365 (t, $6.9 \mathrm{~Hz}$ ), and 36.5 (HSQC) and heteronuclear multiple bond correlation spectroscopy (HMBC) to $58.5 \mathrm{ppm}$, in agreement with literature values of $2.66(\mathrm{t}, 6.9 \mathrm{~Hz}), 58.5$ and 3.35 (t, $6.9 \mathrm{~Hz}$ ), and $36.2 \mathrm{ppm}$ (HMDB00965, accessed from http://www. hmdb.ca/spectra/nmr_one_d/1626 on February 5, 2019). Taurine in $\mathrm{Fmol}^{-/-}$, $2^{-1-}, 4^{-1-}$, and wild-type mouse urine showed the following features: 3.283 (t, $6.6 \mathrm{~Hz}$ ), 50.6 (HSQC) with HMBC to 38.3 and correlated spectroscopy to 3.433 (t, $6.6 \mathrm{~Hz}$ ), and 38.4 (HSQC) and HMBC to $50.5 \mathrm{ppm}$, in agreement with literature values of $3.25(\mathrm{t}, 6.6 \mathrm{~Hz}), 50.4$ and $3.42(\mathrm{t}, 6.6 \mathrm{~Hz})$, and $38.3 \mathrm{ppm}$ (HMDB0000251, accessed from http://www.hmdb.ca/spectra/nmr_one_d/1277 on 5th February 2019). Both hypotaurine and taurine in the urine samples were unambiguously identified using the recent Metabolite Identification Carbon Efficiency (MICE) criteria (Everett, 2015).

\section{Results \\ Fmol $^{-/-}, 2^{-/-}$, and $4^{-/-}$Mice Have a Deficit in Taurine} Production. Previous phenotypic analysis (Hernandez et al., 2009; Shephard and Phillips, 2010; Veeravalli et al., 2014, 2018) has identified metabolic differences between mice in which the Fmo1, Fmo2, and Fmo4 genes had been deleted $\left(\mathrm{Fmol}^{-/-}, 2^{-/-}\right.$, and $4^{-/-}$mice) (Hernandez et al., 2009) and wild-type animals. As an extension of this work, we have used $1 \mathrm{D}{ }^{1} \mathrm{H}$ NMR spectroscopy to compare the urinary metabolite profiles of the knockout-mouse line and wild-type animals. Analysis of the urine of male and female $\mathrm{Fmol}^{-1-}, 2^{-/-}$, and $4^{-1-}$ mice revealed signals at 2.66 and $3.37 \mathrm{ppm}$, corresponding to those of hypotaurine, which were markedly lower in the urine of wild-type mice (Fig. 1, A and B). Signals at 3.28 and 3.43 ppm, corresponding to those of taurine, were detected in the urine of both wild-type and $\mathrm{Fmol}^{-1-}$, $2^{-/-}$, and $4^{-/-}$mice, but their intensities were lower in the latter (Fig. 1, A and B). The identities of taurine and hypotaurine in urine samples were confirmed by two-dimensional NMR (Supplemental Fig. 1). The urinary ratio of taurine to hypotaurine + taurine was significantly less in $\mathrm{Fmol}^{-/-}, 2^{-/-}$, and $4^{-/-}$mice than in wild-type mice $(P<$ 0.0001) (Fig. 1C). In contrast, analysis of male and female $\mathrm{Fmo5}^{-1-}$ mice, in which the gene encoding FMO5 is disrupted (Gonzalez 
A

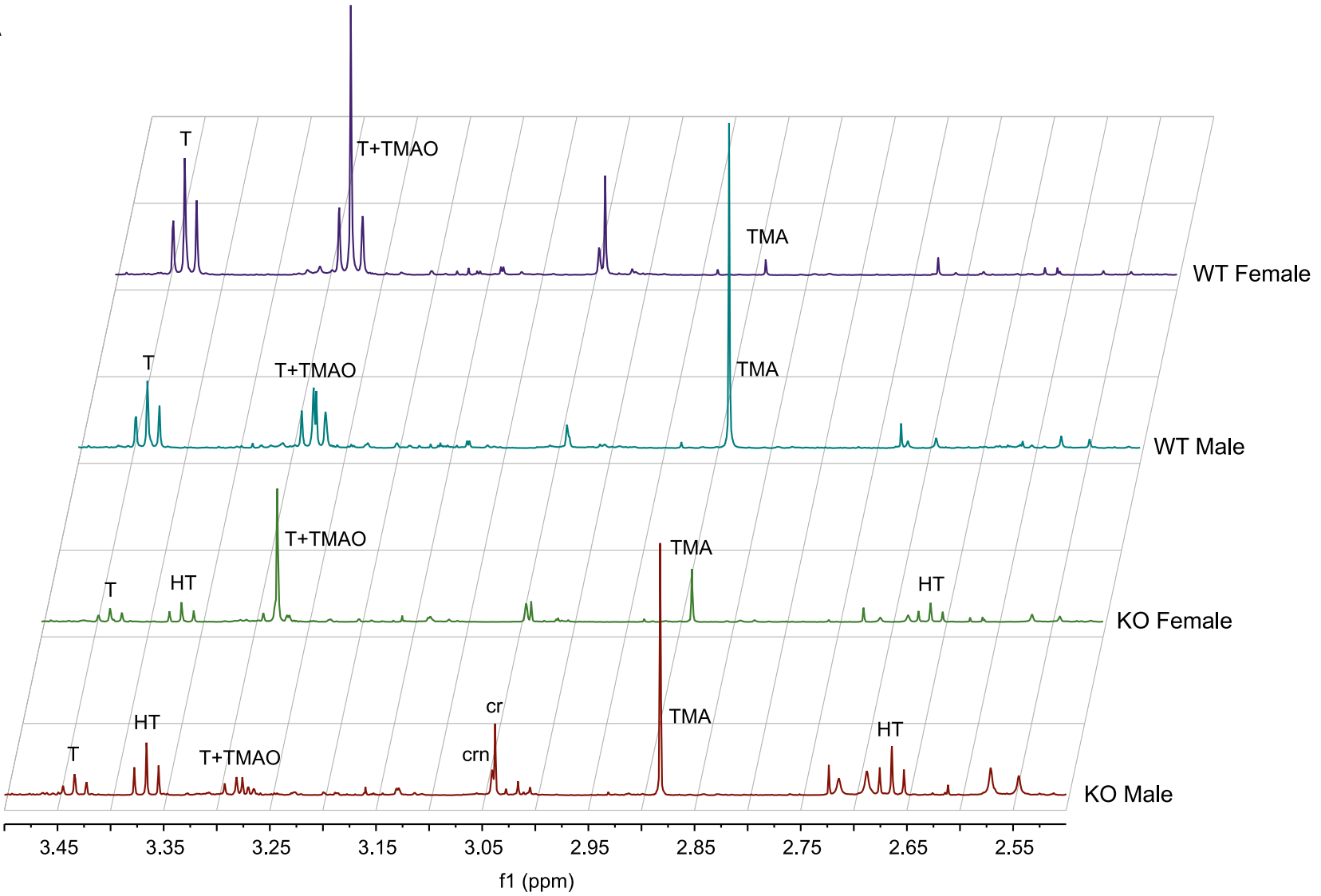

B

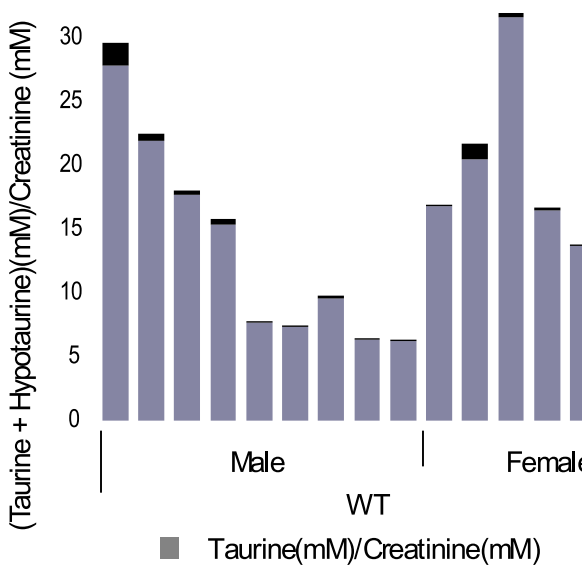

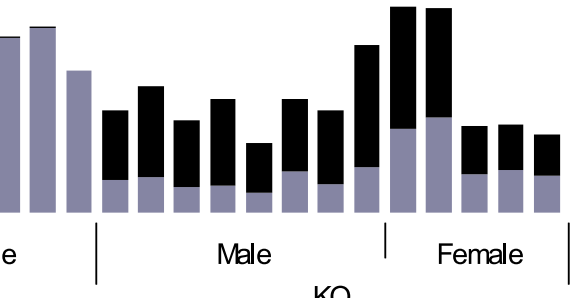

Hypotaurine $(\mathrm{mM}) /$ Creatinine $(\mathrm{mM})$
C

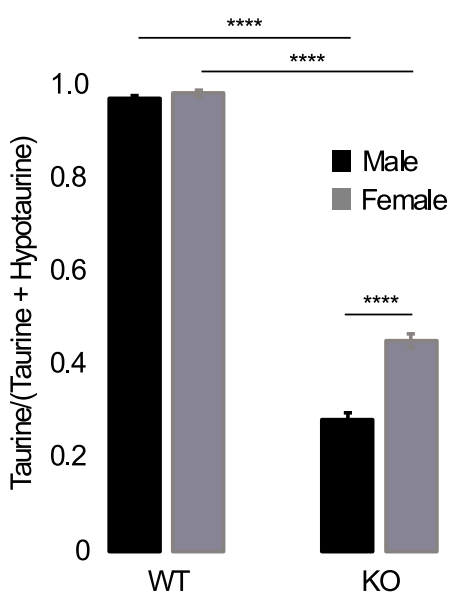

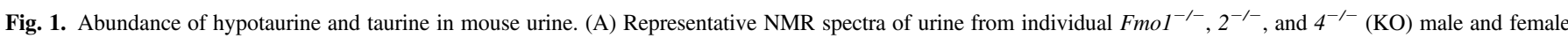

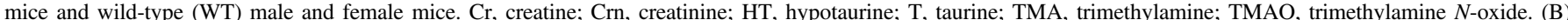

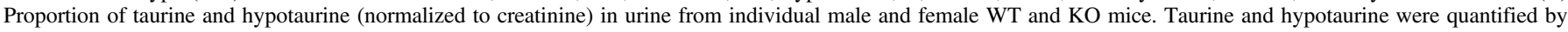

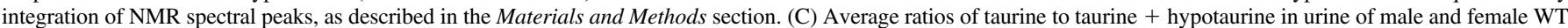

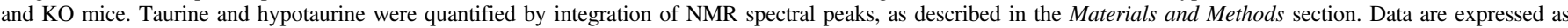
means \pm S.E.M. (WT male, $n=9$; WT female, $n=7$; KO male, $n=8$; KO female, $n=5$ ). $* * * * P<0.0001$.

Malagon et al., 2015), revealed no significant difference from their wildtype counterparts in the urinary ratio of taurine to hypotaurine + taurine (Fig. 2).

Human FMO1 Catalyzes the Oxygenation of Hypotaurine In Vitro. The buildup of hypotaurine and the concomitant decrease of taurine in the urine of $\mathrm{Fmol}^{-/-}, 2^{-/-}$, and $4^{-/-}$mice (Fig. 1) suggests that the formation of taurine from hypotaurine is catalyzed by an FMO. Of the three genes deleted in the $\mathrm{Fmol}^{-1-}, 2^{-/-}$, and $4^{-/-}$knockoutmouse line, the gene encoding FMO4 is expressed at very low levels in mice (Janmohamed et al., 2004) and humans (Dolphin et al., 1996); the gene encoding FMO2 is expressed in low amounts in mice (Janmohamed et al., 2004), and in most humans, the gene does not encode a functional protein because of the presence of a premature stop codon (Dolphin et al., 1998). In contrast, the gene encoding FMO1 is relatively highly expressed in a number of tissues in both mice (Janmohamed et al., 2004) and humans (Dolphin et al., 1996). Therefore, in humans, the most likely candidate for catalyzing the oxygenation of hypotaurine to produce taurine is FMO1. To investigate whether this was the case, 

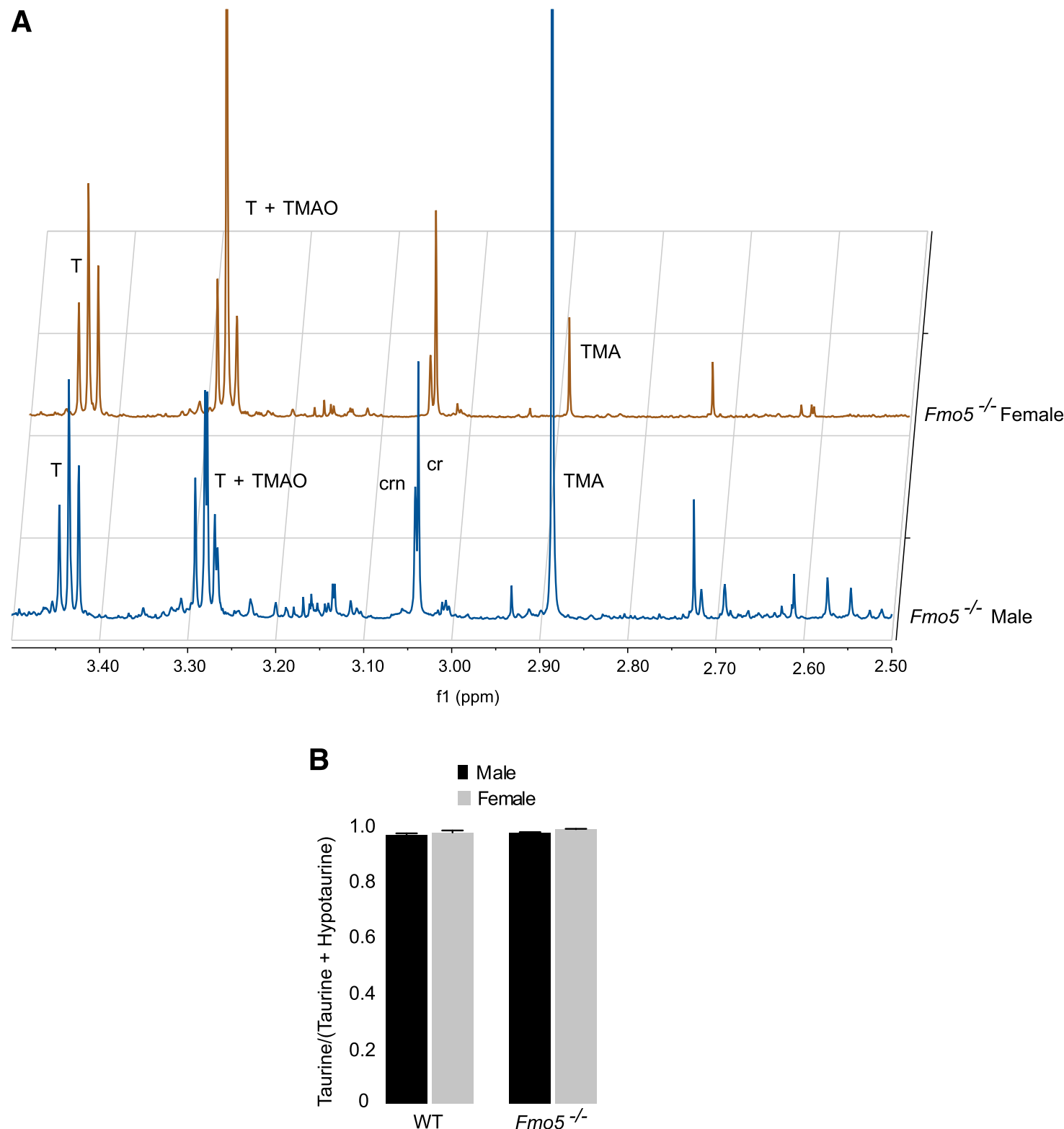

Fig. 2. Analysis of taurine and hypotaurine in the urine of $\mathrm{Fmo5}^{-/-}$mice. (A) Representative NMR spectra of urine from individual $\mathrm{Fmo5^{-/- }}$ male and female mice. Cr, creatine; Crn, creatinine; T, taurine; TMA, trimethylamine; TMAO, trimethylamine $N$-oxide. (B) Average ratios of taurine to taurine + hypotaurine in urine of male and female wild-type (WT) and $\mathrm{Fmo5}^{-/-}$mice. Taurine and hypotaurine were quantified by integration of NMR spectral peaks, as described in the Materials and Methods section. Data are expressed as means \pm S.E.M. (WT male, $n=9$; WT female, $n=7 ; F m o 5^{-1-}$ male, $n=14 ; F m o 5^{-/-}$female, $n=7$ ).

baculosomes containing recombinantly expressed human FMO1 were incubated with hypotaurine and the cofactor NADPH at $\mathrm{pH} 8.5$, the optimum for FMO1, and at the more physiologic $\mathrm{pH}$ of 7.4. Analysis of reaction products by $1 \mathrm{D}{ }^{1} \mathrm{H}$ NMR spectroscopy identified signals at 3.276 and $3.433 \mathrm{ppm}$, corresponding to taurine, at both $\mathrm{pH}$ levels (Fig. 3A). As expected of an FMO1-catalyzed reaction, with NADPH as cofactor, production of taurine was greater at $\mathrm{pH} 8.5$ than at $\mathrm{pH} 7.4(P<$ 0.01 ) (Fig. 3B). In comparison, very little taurine was detected in incubations of control insect cell microsomes at $\mathrm{pH} 8.5$ and none at $\mathrm{pH}$ 7.4 (Fig. 3, A and B). The identity of taurine as a product of FMO1catalyzed reactions was confirmed by high-resolution ultra-performance liquid chromatography electrospray mass spectrometry against an authentic reference standard (Supplemental Figs. 2 and 3) (Dona et al., 2016).

We also investigated whether the conversion of hypotaurine to taurine could be catalyzed by either of the two other major functional FMOs of humans, FMO3 and FMO5 (Overby et al., 1997). In comparison with baculosomes containing human FMO1, those containing FMO3 produced much lower amounts of taurine at $\mathrm{pH} 8.5$ or 7.4 , with either $\mathrm{NADPH}$ or NADH as cofactor $(P<0.01)$. As expected from the analysis of the urine of $\mathrm{Fmo5}^{-/-}$mice (Fig. 2), production of taurine by baculosomes containing human FMO5 was similar to that produced by control insect cell microsomes (Fig. 3B). These results confirm that production of taurine from hypotaurine can be catalyzed by FMO1 and, to a much smaller extent, by FMO3 but not significantly by FMO5.

The production of taurine in the Fmol-null mice was less affected in females $(\sim 55 \%$ depletion) than in males $(\sim 70 \%$ depletion) $(P<$ 0.0001 ) (Fig. 1C). This gender difference is likely due to the contribution in female Fmol-null mice of FMO3, which is absent from the liver of adult male mice (Falls et al., 1995; Janmohamed et al., 2004). In the case of wild-type mice, the presence of FMO1, which is more effective than 


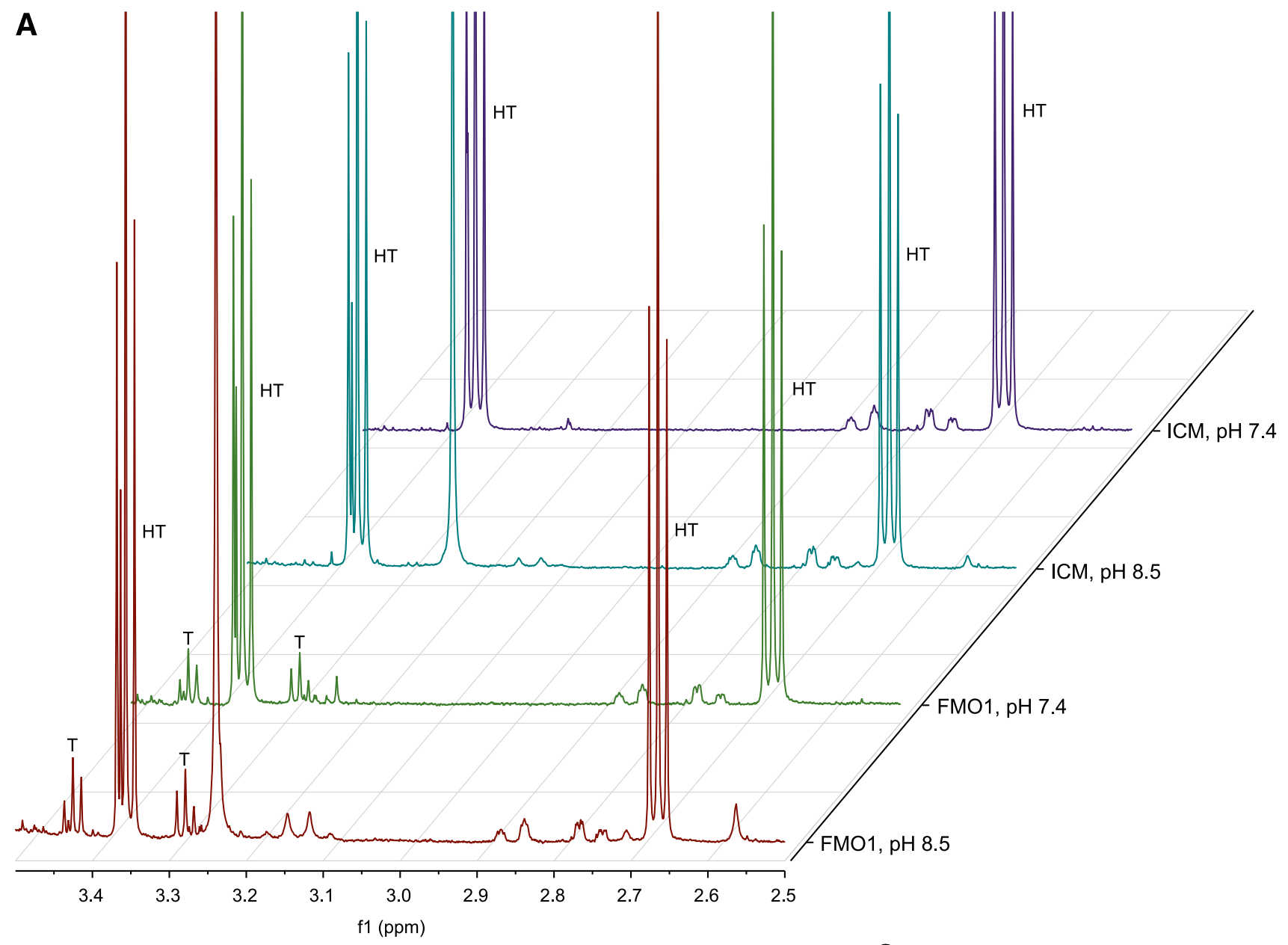

B
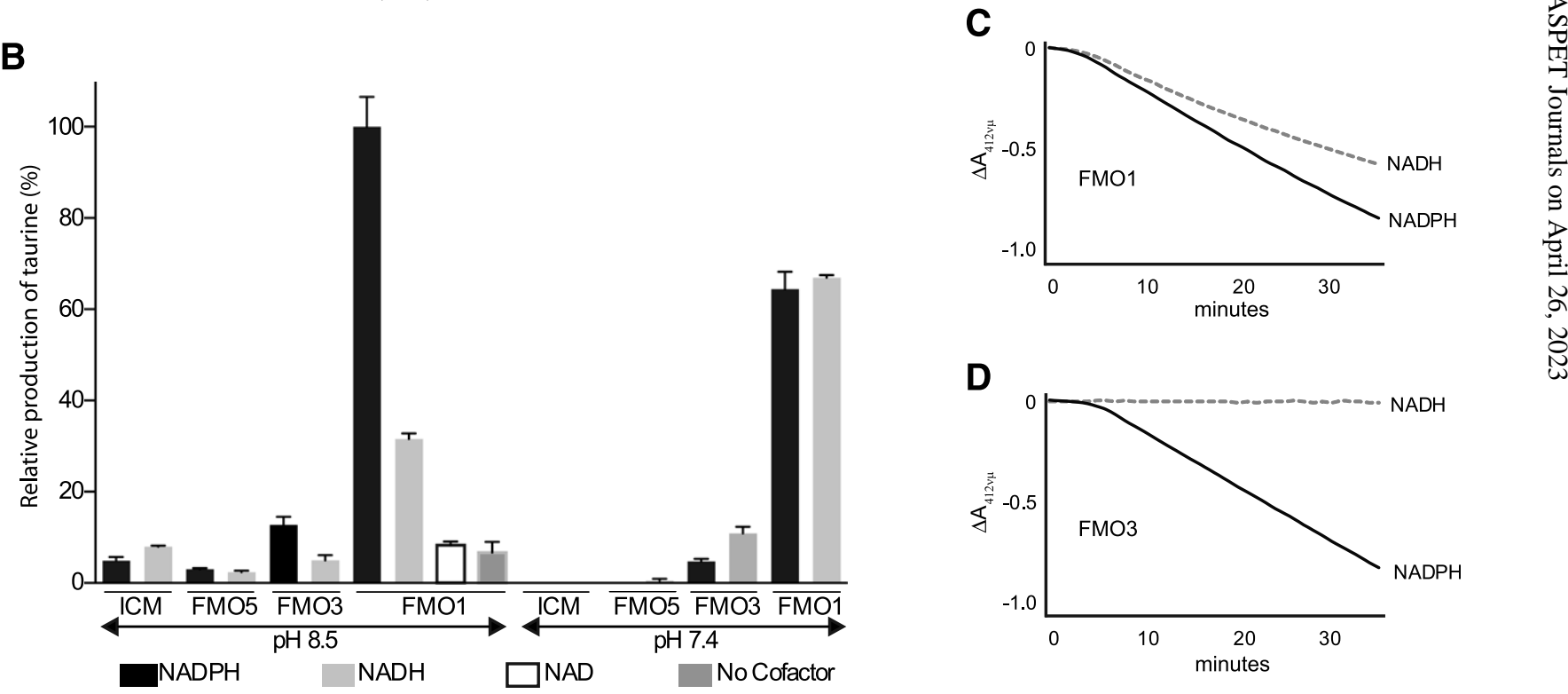

Fig. 3. Analysis of in vitro enzyme-catalyzed reactions. (A) Representative NMR spectra of products of reactions catalyzed by baculosomes containing human FMO1 or by insect cell microsomes (ICM) at pH 8.5 or 7.4. Note: the singlet signal at ca $3.364 \mathrm{ppm}$ overlapping the triplet because of hypotaurine is due to methanol. HT, hypotaurine; T, taurine. (B) Production of taurine from hypotaurine in reactions catalyzed by human FMO1, FMO3, or FMO5 or by ICM at pH 8.5 or 7.4 with NADPH or NADH as cofactor. In the case of reactions catalyzed by FMO1 at pH 8.5, NAD or no cofactor were also used. Taurine production was quantified by integration of NMR spectral peaks and is plotted relative to that produced by FMO1-containing baculosomes at $\mathrm{pH} 8.5$ with NADPH as cofactor (set at $100 \%)$. Data are expressed as means $\pm 0.5 \times$ range $(n=$ 2). (C and D) Representative progress curves of methimazole $S$-oxygenation catalyzed by human FMO1 (C) or FMO3 (D), at pH 8.5, with NADPH or NADH as cofactor. Reactions were monitored at $\mathrm{A}_{412} \mathrm{~nm}$. 
A

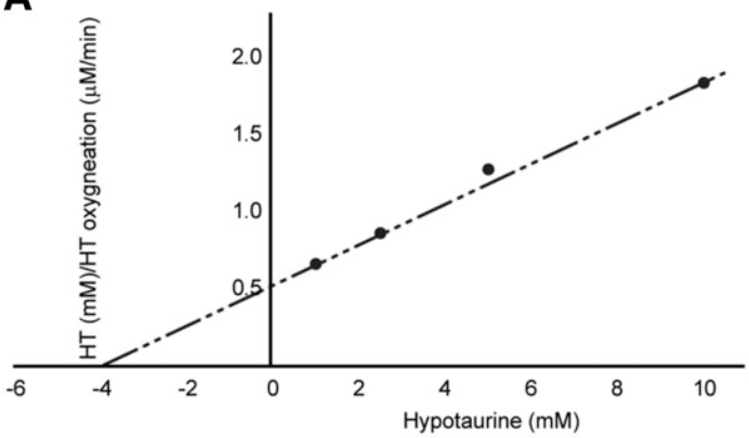

B

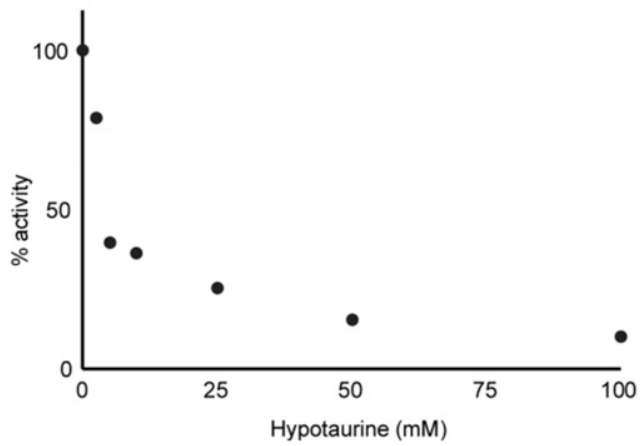

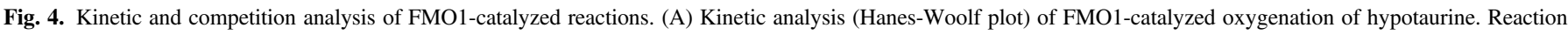

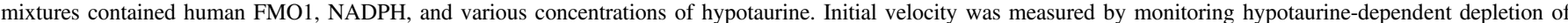

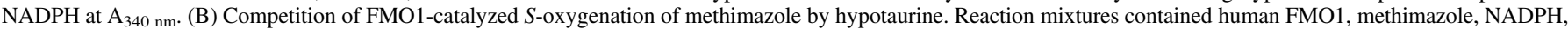

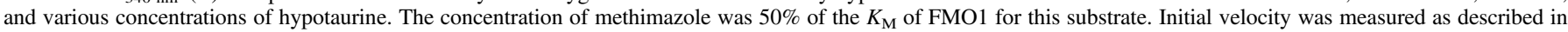
Materials and Methods. Methimazole S-oxygenation activity was plotted as a percentage of that in the absence of hypotaurine.

FMO3 in catalyzing the formation of taurine from hypotaurine as evidenced by analysis in vitro (Fig. 3B), greatly outweighs the effect of the presence in females of FMO3.

Human FMO1 Can Use Both NADPH and NADH as Cofactors. FMOs, despite being termed NADPH-dependent monooxygenases, have been reported to be able also to use NADH as a cofactor (Hvattum et al., 1991). We found that in catalyzing the production of taurine from hypotaurine, FMO1 could use either NADPH or NADH as cofactor (Fig. 3B). However, whereas NADPH is the more effective cofactor at $\mathrm{pH} 8.5(P<0.01)$, the cofactors are equally effective at $\mathrm{pH} 7.4$ (Fig. 3B). When NAD was used as cofactor for FMO1, the amount of taurine produced was very low and not significantly different from that produced in the absence of cofactor (Fig. 3B). We therefore investigated whether FMO1 could use NADH as a cofactor for the $S$-oxygenation of other substrates. NADH was an effective cofactor for the FMO1catalyzed $S$-oxygenation of the FMO model substrate methimazole (Fig. 3C). However, in the case of FMO3, NADH was ineffective as a cofactor for methimazole oxygenation, which was dependent on NADPH (Fig. 3D).

Kinetic Parameters of FMO1-Catalyzed $S$-Oxygenation of Hypotaurine. Kinetic parameters of FMO1-catalyzed oxygenation of hypotaurine to produce taurine were estimated under conditions at which the enzyme was most active: $\mathrm{pH} 8.5$ with NADPH as cofactor (Fig. 3B). Simultaneous measurement of NADPH signal decrease and taurine signal increase in ${ }^{1} \mathrm{H}$ NMR spectra revealed that under these conditions, there was a 1:1 ratio of taurine production to NADPH oxidation. The kinetics of the enzyme-catalyzed reaction could therefore be assessed by monitoring depletion of NADPH (measured at $\mathrm{A}_{340 \mathrm{~nm}}$ ). A plot of kinetic data are shown in Fig. 4A. Direct linear plots of data (velocity vs. substrate concentration) in parameter space (Cornish-Bowden and Wharton, 1988) gave estimates for $K_{\mathrm{M}}$ of $\sim 4.1 \mathrm{mM}$ and $\mathrm{V}_{\max }$ of $\sim 7.5 \mu \mathrm{M}$ minute ${ }^{-1}$, giving a catalytic constant $\left(k_{\text {cat }}\right)$ of $\sim 55$ minutes $^{-1}$. Further support for the ability of FMO1 to use hypotaurine as a substrate is provided by the finding that hypotaurine, at concentrations comparable with the $K_{\mathrm{M}}$ of FMO1-catalyzed hypotaurine oxygenation, acted as an effective competitor of FMO1-catalyzed $S$-oxygenation of the model FMO substrate methimazole (Fig. 4B).

\section{Discussion}

We have confirmed both in vivo by ${ }^{1} \mathrm{H}$ NMR metabolite profiling of the urine of $\mathrm{Fmol}^{-/-}, 2^{-/-}$, and $4^{-/-}$mice and in vitro by analysis of the catalytic activity of human FMOs that formation of taurine from hypotaurine is catalyzed by FMO1, a monooxygenase, and that the enzyme can use either NADPH or NADH as cofactor for the $S$-oxygenation reaction. FMO1 is also able to use NADPH or NADH to catalyze the $S$-oxygenation of methimazole. It is not known whether the ability of the enzyme to use NADH as cofactor extends to $\mathrm{N}$-oxygenation reactions.

Our results from $\mathrm{Fmol}^{-/-}, 2^{-/-}$, and $4^{-/-}$mice show that in the absence of FMO1, most taurine production is abolished in both males and females, suggesting that, in mice, the major source of this abundant amino acid is the FMO1-catalyzed oxygenation of hypotaurine (Fig. 5). Consistent with this, the lack of production of taurine from hypotaurine in vitro in the absence of enzyme indicates that nonenzymatic conversion does not contribute substantially to taurine production.

FMOs (EC 1.14.13.8) are best known for their role in the metabolism of xenobiotics, including therapeutic drugs (reviewed in Phillips and Shephard, 2017) and foreign chemicals such as organophosphate insecticides (reviewed in Krueger and Williams, 2005). Of the members of the FMO family, FMO1 has the broadest substrate range (reviewed in Krueger and Williams, 2005). In addition to its role in xenobiotic metabolism, FMO1 has been identified as a novel regulator of energy balance (Veeravalli et al., 2014).

As taurine can be obtained from the diet, the relative contribution of FMO1-catalyzed monooxygenation of hypotaurine to the amount of taurine in humans is not known. In human liver, FMO1 is expressed in the fetus but is switched off after birth (Dolphin et al., 1991, 1996; Koukouritaki et al., 2002). This pattern of expression is consistent with the decline in taurine concentration in human liver after birth (Sturman and Gaull, 1975). In contrast, in adult rodent liver, the gene encoding FMO1 is highly expressed (Janmohamed et al., 2004) and taurine is abundant (Oja and Kontro, 1981). In adult humans, although FMO1 is not expressed in liver, it is expressed in a range of extra-hepatic tissues in which the action of taurine has been implicated, such as kidney (Dolphin et al., 1996), brain (Hernandez et al., 2004), small intestine (Yeung et al., 2000), and heart (Kim et al., 2003) as well as a number of endocrine tissues, including pancreas, adrenal, and testis (Hernandez et al., 2004).

Concentrations of taurine are high in fetal brain but fall during development (Sturman and Gaull, 1975). The decline in taurine concentration in the developing brain is consistent with the decrease in expression of Fmol in the mouse brain during development (Janmohamed et al., 2004). FMO1 protein is active in the mouse brain, as evidenced by its catalysis of the $N$-oxygenation of the tricyclic antidepressant imipramine (Hernandez et al., 2009). FMO1 would therefore be expected to contribute to the production in brain of taurine from the precursor hypotaurine. 
<smiles>NC(CS)C(=O)O</smiles>

Cysteine<smiles>CC(C)C(=O)O</smiles><smiles>NC(CS(=O)O)C(=O)O</smiles>

Cysteine sulfinate<smiles>C[C@H](CCN)C(=O)O</smiles>

Hypotaurine<smiles>NCCS(=O)(=O)O</smiles>

Taurine

Fig. 5. Terminal steps of the biosynthetic pathway of taurine from cysteine. The final reaction in the pathway is catalyzed by FMO1, using either NADPH or NADH as cofactor. CDO, cysteine dioxygenase; CSD, cysteine sulfinate decarboxylase; FMO1, flavin-containing monooxygenase 1 .

Taurine deficiency is implicated in a number of pathologic conditions, including cardiomyopathy, muscular abnormalities, and renal dysfunction (Ripps and Shen, 2012). Conversely, taurine supplementation has been reported to have positive effects on health, for instance, in lowering total plasma cholesterol (Murakami, 2015) and in overcoming insulin resistance (Ripps and Shen, 2012). Given our finding that FMO1 catalyzes the formation of taurine from hypotaurine, it is of interest that Fmol-null mice exhibit some characteristics in common with those of taurine deficiency, including raised plasma concentrations of cholesterol (Veeravalli et al., 2018) and glucose (Veeravalli et al., 2014).

The $K_{\mathrm{M}}$ of FMO1 for hypotaurine is similar to that of human cysteine dioxygenase, an upstream enzyme in the taurine biosynthetic pathway (Fig. 5), for its substrate cysteine (Ye et al., 2007). Although the $K_{\mathrm{M}}$ for the FMO1-catalyzed oxygenation of hypotaurine is high, our results from the knockout-mouse line indicate that FMO1 is physiologically relevant for the production of taurine.
In addition to Fmol, two other genes are deleted in the knockoutmouse line, Fmo2 and Fmo4. Most humans are homozygous for a nonsense mutation of $F M O 2$, c. $1414 \mathrm{C}>\mathrm{T}[\mathrm{p} .(\mathrm{Gln} 472 *)]$, the $F M O 2 * 2 A$ allele, and do not express functional FMO2 (Dolphin et al., 1998; Whetstine et al., 2000); in the case of FMO4, the gene is expressed at very low levels (Dolphin et al., 1996). Thus, although we cannot eliminate the possibility that FMO2 or FMO4 are able to catalyze hypotaurine oxygenation in vitro, neither of these enzymes is likely to contribute substantially to the production of taurine in humans in vivo.

Commensurate with a role for FMO1 in endogenous metabolism, the gene contains few nonsynonymous polymorphisms (Phillips et al., 2007), each of which is present at very low frequency (Furnes et al., 2003), and only one has a significant but substrate-dependent effect on catalytic activity (Furnes and Schlenk, 2004). However, interindividual variation of up to fivefold in the expression of FMO1 in adult human tissues such as kidney (Krause et al., 2003) and small intestine (Yeung et al., 2000) could affect taurine production and thus contribute to an intracellular deficiency of the amino acid.

\section{Acknowledgments}

We thank Mohamed Said and Dorna Varshavi for assistance with NMR spectroscopy and Dr. Iain Goodall for assistance with ultra-performanc liquid chromatography-mass spectrometry. We also thank Jeremy Nicholson and Elaine Holmes for access to NMR facilities at Imperial College London.

\section{Authorship Contributions}

Participated in research design: Veeravalli, Phillips, Freire, Everett, Shephard.

Conducted experiments: Veeravalli, Phillips, Freire, Varshavi, Everett, Shephard.

Contributed new reagents or analytic tools: Veeravalli, Phillips, Freire, Varshavi, Everett, Shephard.

Performed data analysis: Veeravalli, Phillips, Freire, Varshavi, Everett, Shephard.

Wrote or contributed to the writing of the manuscript: Phillips, Everett, Shephard.

\section{References}

Aruoma OI, Halliwell B, Hoey BM, and Butler J (1988) The antioxidant action of taurine, hypotaurine and their metabolic precursors. Biochem J 256:251-255.

Cavallini D, De Marco C, Mondovi B, and Stirpe F (1954) The biological oxidation of hypotaurine. Biochim Biophys Acta 15:301-303.

Cornish-Bowden A and Wharton CW (1988) Enzyme Kinetics, IRL Press Ltd., Oxford.

Dixit A and Roche TE (1984) Spectrophotometric assay of the flavin-containing monooxygenase and changes in its activity in female mouse liver with nutritional and diurnal conditions. Arch Biochem Biophys 233.50-63.

Dolphin C, Shephard EA, Povey S, Palmer CNA, Ziegler DM, Ayesh R, Smith RL, and Phillips IR (1991) Cloning, primary sequence, and chromosomal mapping of a human flavin-containing monooxygenase (FMO1). J Biol Chem 266:12379-12385.

Dolphin CT, Beckett DJ, Janmohamed A, Cullingford TE, Smith RL, Shephard EA, and Phillips IR (1998) The flavin-containing monooxygenase 2 gene (FMO2) of humans, but not of other primates, encodes a truncated, nonfunctional protein. J Biol Chem 273:30599-30607.

Dolphin CT, Cullingford TE, Shephard EA, Smith RL, and Phillips IR (1996) Differential developmental and tissue-specific regulation of expression of the genes encoding three members of the flavin-containing monooxygenase family of man, FMO1, FMO3 and FM04. Eur J Biochem 235:683-689.

Dona AC, Kyriakides M, Scott F, Shephard EA, Varshavi D, Veselkov K, and Everett JR (2016) A guide to the identification of metabolites in NMR-based metabonomics/metabolomics experiments. Comput Struct Biotechnol J 14:135-153.

Edgar SE, Hickman MA, Marsden MM, Morris JG, and Rogers QR (1994) Dietary cysteic acid serves as a precursor of taurine for cats. $J$ Nutr 124:103-109.

Everett JR (2015) A new paradigm for known metabolite identification in metabonomics/metabolomics: metabolite identification efficiency. Comput Struct Biotechnol J 13:131-144.

Falls JG, Blake BL, Cao Y, Levi PE, and Hodgson E (1995) Gender differences in hepatic expression of flavin-containing monooxygenase isoforms (FMO1, FMO3, and FMO5) in mice. J Biochem Toxicol 10:171-177.

Furnes B, Feng J, Sommer SS, and Schlenk D (2003) Identification of novel variants of the flavincontaining monooxygenase gene family in African Americans. Drug Metab Dispos 31:187-193.

Furnes B and Schlenk D (2004) Evaluation of xenobiotic N- and S-oxidation by variant flavincontaining monooxygenase 1 (FMO1) enzymes. Toxicol Sci 78:196-203.

Gonzalez Malagon SG, Melidoni AN, Hernandez D, Omar BA, Houseman L, Veeravalli S, Scott F, Varshavi D, Everett J, Tsuchiya Y, et al. (2015) The phenotype of a knockout mouse identifies flavin-containing monooxygenase 5 (FMO5) as a regulator of metabolic ageing. Biochem Pharmacol 96:267-277. 
Haug K, Salek RM, Conesa P, Hastings J, de Matos P, Rijnbeek M, Mahendraker T, Williams M, Neumann S, Rocca-Serra P, et al. (2013) MetaboLights--an open-access general-purpose repository for metabolomics studies and associated meta-data. Nucleic Acids Res 41:D781-D786.

Hernandez D, Chandan P, Janmohamed A, Phillips IR, and Shephard EA (2006a) Deletion of genes from the mouse genome using Cre/loxP technology. Methods Mol Biol 320:307-319.

Hernandez D, Janmohamed A, Chandan P, Omar BA, Phillips IR, and Shephard EA (2009) Deletion of the mouse Fmol gene results in enhanced pharmacological behavioural responses to imipramine. Pharmacogenet Genomics 19:289-299.

Hernandez D, Janmohamed A, Chandan P, Phillips IR, and Shephard EA (2004) Organization and evolution of the flavin-containing monooxygenase genes of human and mouse: identification of novel gene and pseudogene clusters. Pharmacogenetics 14:117-130.

Hernandez D, Melidoni AN, Phillips IR, and Shephard EA (2006b) Microinjection of targeted embryonic stem cells and establishment of knockout mouse lines for Fmo genes. Methods Mo Biol 320:329-341.

Huxtable RJ (1992) Physiological actions of taurine. Physiol Rev 72:101-163.

Hvattum E, Bergseth S, Pedersen CN, Bremer J, Aarsland A, and Berge RK (1991) Microsomal oxidation of dodecylthioacetic acid (a 3-thia fatty acid) in rat liver. Biochem Pharmacol 41: 945-953.

Janmohamed A, Hernandez D, Phillips IR, and Shephard EA (2004) Cell-, tissue-, sex- and developmental stage-specific expression of mouse flavin-containing monooxygenases (Fmos). Biochem Pharmacol 68:73-83.

Kim YH, Lim DS, Lee JH, Shim WJ, Ro YM, Park GH, Becker KG, Cho-Chung YS, and Kim MK (2003) Gene expression profiling of oxidative stress on atrial fibrillation in humans. Exp Mo Med 35:336-349.

Koukouritaki SB, Simpson P, Yeung CK, Rettie AE, and Hines RN (2002) Human hepatic flavincontaining monooxygenases 1 (FMO1) and 3 (FMO3) developmental expression. Pediatr Res 51:236-243.

Krause RJ, Lash LH, and Elfarra AA (2003) Human kidney flavin-containing monooxygenases and their potential roles in cysteine s-conjugate metabolism and nephrotoxicity. $J$ Pharmacol Exp Ther 304:185-191.

Krueger SK and Williams DE (2005) Mammalian flavin-containing monooxygenases: structure/ function, genetic polymorphisms and role in drug metabolism. Pharmacol Ther 106:357-387.

Lombardini JB (1983) Effects of ATP and taurine on calcium uptake by membrane preparations of the rat retina. $J$ Neurochem 40:402-406.

Murakami S (2015) Role of taurine in the pathogenesis of obesity. Mol Nutr Food Res 59:1353-1363.

Oja SS and Kontro P (1981) Oxidation of hypotaurine in vitro by mouse liver and brain tissues. Biochim Biophys Acta 677:350-357.

Overby LH, Carver GC, and Philpot RM (1997) Quantitation and kinetic properties of hepatic microsomal and recombinant flavin-containing monooxygenases 3 and 5 from humans. Chem Biol Interact 106:29-45.

Phillips IR, Francois AA, and Shephard EA (2007) The flavin-containing monoooxygenases (FMOs): genetic variation and its consequences for the metabolism of therapeutic drugs. Curr Pharmacogenom 5:292-313.

Phillips IR and Shephard EA (2017) Drug metabolism by flavin-containing monooxygenases of human and mouse. Expert Opin Drug Metab Toxicol 13:167-181.
Ripps H and Shen W (2012) Review: taurine: a "very essential" amino acid. Mol Vis 18: 2673-2686.

Shephard EA and Phillips IR (2010) The potential of knockout mouse lines in defining the role of flavin-containing monooxygenases in drug metabolism. Expert Opin Drug Metab Toxicol 6: 1083-1094.

Sturman JA and Gaull GE (1975) Taurine in the brain and liver of the developing human and monkey. J Neurochem 25:831-835.

Sumizu K (1962) Oxidation of hypotaurine in rat liver. Biochim Biophys Acta 63:210-212.

Varshavi D, Scott FH, Varshavi D, Veeravalli S, Phillips IR, Veselkov K, Strittmatter N, Takats Z, Shephard EA, and Everett JR (2018) Metabolic biomarkers of ageing in C57bl/6J wild-type and flavin-containing monooxygenase 5 (FMO5)-knockout mice. Front Mol Biosci 5:28.

Veeravalli S, Karu K, Scott F, Fennema D, Phillips IR, and Shephard EA (2018) Effect of flavincontaining monooxygenase genotype, mouse strain, and gender on trimethylamine N-oxide production, plasma cholesterol concentration, and an index of atherosclerosis. Drug Metab Dispos 46:20-25.

Veeravalli S, Omar BA, Houseman L, Hancock M, Gonzalez Malagon SG, Scott F, Janmohamed A, Phillips IR, and Shephard EA (2014) The phenotype of a flavin-containing monooyxgenase knockout mouse implicates the drug-metabolizing enzyme FMO1 as a novel regulator of energy balance. Biochem Pharmacol 90:88-95.

Whetstine JR, Yueh M-F, McCarver DG, Williams DE, Park CS, Kang JH, Cha YN, Dolphin CT, Shephard EA, Phillips IR, et al. (2000) Ethnic differences in human flavin-containing monooxygenase 2 (FMO2) polymorphisms: detection of expressed protein in African-Americans. Toxicol Appl Pharmacol 168:216-224.

Wishart DS, Feunang YD, Marcu A, Guo AC, Liang K, Vázquez-Fresno R, Sajed T, Johnson D, Li C, Karu N, et al. (2018) HMDB 4.0: the human metabolome database for 2018. Nucleic Acids Res 46 (D1):D608-D617.

Yancey PH (2005) Organic osmolytes as compatible, metabolic and counteracting cytoprotectants in high osmolarity and other stresses. $J$ Exp Biol 208:2819-2830.

Ye S, Wu X, Wei L, Tang D, Sun P, Bartlam M, and Rao Z (2007) An insight into the mechanism of human cysteine dioxygenase. Key roles of the thioether-bonded tyrosine-cysteine cofactor. J Biol Chem 282:3391-3402.

Yeung CK, Lang DH, Thummel KE, and Rettie AE (2000) Immunoquantitation of FMO1 in human liver, kidney, and intestine. Drug Metab Dispos 28:1107-1111.

Address correspondence to: Elizabeth A. Shephard, Department of Structural and Molecular Biology, University College London, London WC1E 6BT, UK. E-mail: e.shephard@ucl.ac.uk;lan R. Phillips, Department of Structural and Molecular Biology, University College London, London WC1E 6BT, UK. E-mail: i.r.phillips@ ucl.ac.uk; or Jeremy R. Everett, Medway Metabonomics Research Group, University of Greenwich, Chatham Maritime, Kent ME4 4TB, UK. E-mail: j.r. everett@greenwich.ac.uk 